\title{
Latest Results on Weak Semi-Leptonic Hyperon Decays in KTeV
}

\author{
Nickolas Solomey ${ }^{\mathrm{a}}$ \\ aEnrico Fermi Institute, The University of Chicago, Chicago, IL 60637, USA
}

The KTeV experiment at Fermilab has finished its $\Xi^{0}$ semi-leptonic decay analysis from the 1997 run and presented here are the final numbers for the branching ratio and form factors. A new run with three times the statistics was taken in 1999 and a first look at the data shows the quality and consistency of the results, predicting a similar improvement in these measurements.

\section{Semi-Leptonic $\Xi^{0}$ Decays}

Semi-leptonic hyperon decays are important as tests for new physics by measuring their form factors and branching ratios [1. This work started experimentally with $\Lambda$ beta decay [2, 3]:

$\Lambda \rightarrow p e^{-} \bar{\nu}$.

It was followed by a high statistics sample of $\Sigma^{-}$ beta decays [4:

$\Sigma^{-} \rightarrow n e^{-} \bar{\nu}$

and $\Xi^{-}$beta decays $[5$ :

$\Xi^{-} \rightarrow \Lambda e^{-} \bar{\nu} \quad$ and $\quad \Xi^{-} \rightarrow \Sigma^{0} e^{-} \bar{\nu}$.

It was proposed in 1993 to extend the $\mathrm{KTeV}$ program $[6]$ to include a search for the unseen $\Xi^{0}$ beta decays $[7$ :

$\Xi^{0} \rightarrow \Sigma^{+} e^{-} \bar{\nu}$

This suggestion of the author was shown to be accessible in $\mathrm{KTeV}$ because even though there was no charged particle vertex, the $\pi^{0}$ decay into two gammas and the high precision electronmagnetic calorimeter permits the vertex to be precisely measured by constraining to the $\pi^{0}$ mass [8]. This along with the high flux of $\Xi^{0}$ hyperons in the experiment permitted these analysis.

Hyperon semi-leptonic decays with a muon replacing the electron are only energetically permitted for a few decay modes. In all there were only three types of such decays ever observed and all of these with a very small number of events. It was the author who introduced and performed the analysis in $\mathrm{KTeV}$ of the $\Xi^{0}$ muonic decay [9]. These are important because they can be a first glimpse at the $\mathrm{g}_{3}$ form factor which is multiplied by $\left(\mathrm{M}_{\text {lepton }} / \mathrm{M}_{\text {hyperon }}\right)$; in beta decays this ratio squelches any effect of the $g_{3}$ form factor, but in muonic decays this term, although small, is no longer negligible [10].

\section{Final Results from $\mathrm{KTeV}-97$}

Many summary talks of the $\mathrm{KTeV}$ hyperon data and analysis techniques are available elsewhere 111. The goal of this section is to summarize in one place the final numbers available at this time. A Physical Review Letter was put out with a fraction of the KTeV 1997 data called the winter run, yielding a branching ratio based on 176 events of $2.71( \pm 0.22 \pm 0.31) \times 10^{-4}$ [12], where the first error is statistical and the second systematic. Since then the remaining 1997 data sample has been analyzed 13] and the branching ratio for the $\Xi^{0}$ beta decay from this separate sample is $2.60( \pm 0.11 \pm 0.16) \times 10^{-4}$, based on 626 events. The theoretical expected is $2.6 \times 10^{-4}$. The $\Xi^{0}$ muonic decay has a preliminary branching ratio of $3.5\left(\begin{array}{ll}+2.0 & +0.5 \\ -1.0\end{array}\right) \times 10^{-6}$ based on 5 events, again the first error is statistical and the second systematic the asymmetric errors are due to the small sample of events; the theoretical expected is $2.6 \times 10^{-6}$.

The final four form factors obtained in our analysis 114] using a ultra clean sample of $\Xi^{0}$ beta decays by using the added power of the TRD particle identification [15] are: $f_{1}=0.99 \pm 0.14, \frac{g_{1}}{f_{1}}=$ $1.12 \pm 0.27, \frac{f_{2}}{f_{1}}=2.3 \pm 1.3$, and $\frac{g_{2}}{f_{1}}=-1.4 \pm 2.1$ 


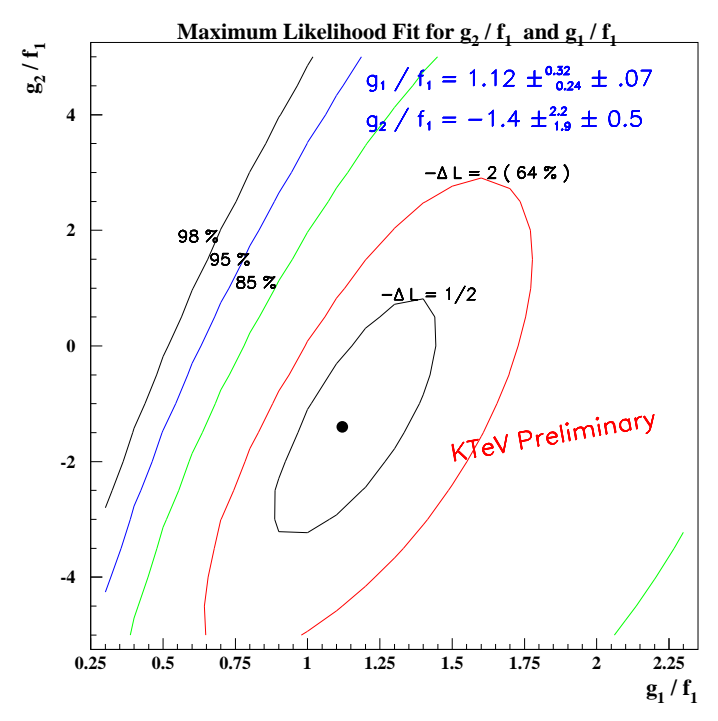

Figure 1. Maximum likelihood fit of the form factors $\mathrm{g}_{1}$ and $\mathrm{g}_{2}$, the later is consistent with zero.

(the errors are combined statistical and systematic for brevity); here this analysis used the previously quoted branching ratio, and permitted the $g_{2}$ form factor to float. The $g_{2}$ form factor is consistent with zero, see figure 1, and in another analysis it was constrained to be zero and the remaining three form factors reanalyzed and they essentially remained unchanged.

\section{Improved Form Factors}

The form factors reported in the previous section are also dependent upon the mass of the $\Xi^{0}$ particle and its life time. If either of these numbers are off then this can have an effect upon the form factor results. The values used were those from the $1998 \mathrm{PDG}$ [16]. The $\mathrm{KTeV}$ experiment has tried improving both of these values: a first attempt at improving the mass was made [17], but another experiment 18 has released an improved value before our measurement was finished and they have an error that is better than what

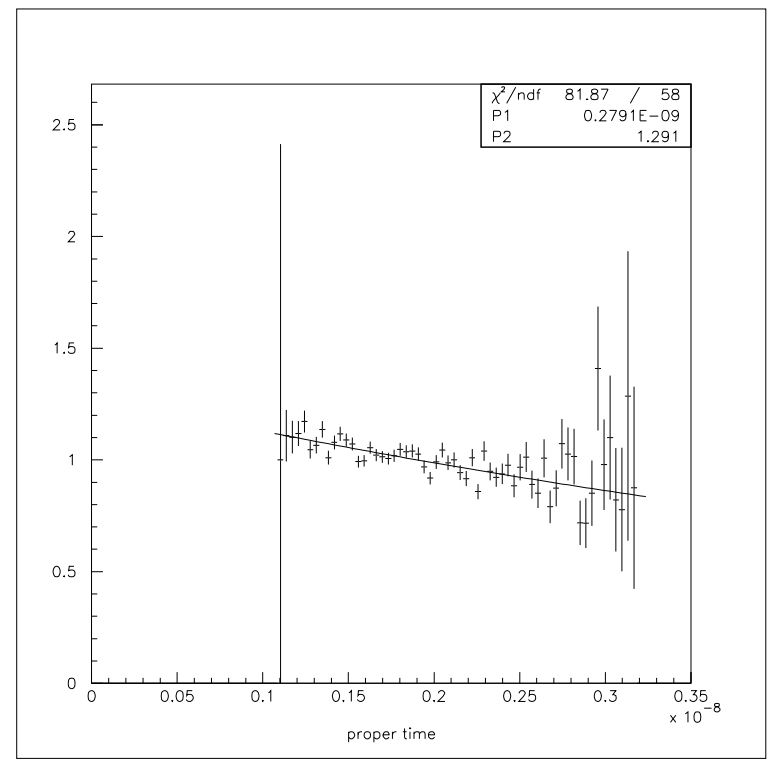

Figure 2. Ratio of data and normalized MonteCarlo of the proper time (in seconds) of the decay $\Xi^{0} \rightarrow \Lambda^{0} \pi^{0}$. Our preliminary lifetime measurement is $2.79( \pm 0.01 \pm 0.05) \times 10^{-10}$ seconds, the first error is statistical based on a fit to 94839 events, the second is the systematic error from $\mathrm{z}$ and $\mathrm{p}_{\Xi^{0}}$ dependency.

we would have achieved so this analysis was not pursued; however, we have made a factor of three improvement in the life time of the $\Xi^{0}$ [19], see figure2. Reevaluation of the form factors with these two improved values will lead to slightly different values, but consistent with the error bars.

An accurate determination of the $\Xi^{0}$ mass is also important as a test of $\mathrm{SU}(3)$ symmetry in the Coleman-Glashow mass relationship between the hyperons:

$M_{\Xi^{-}}-M_{\Xi^{0}}=\left(M_{p}-M_{n}\right)+\left(M_{\Sigma^{-}}-M_{\Sigma^{+}}\right)$

Using the new mass value, yet to be incorporated in the PDG, $1314.82 \pm 0.06$, this equates to $6.49 \pm 0.14=6.73 \pm 0.08$, a small $\mathrm{SU}(3)$ symmetry breaking less than $3.6 \%$. However, there is a hyperon beta decay that is yet unseen that is an 
even better test:

$\Xi^{-} \rightarrow \Xi^{0} e^{-} \bar{\nu}$

This is such a powerful test of $\mathrm{SU}(3)$ symmetry breaking because there is only $\sim 6.49 \mathrm{MeV}$ of energy being released in this beta decay, so by measuring the branching ratio this is the most accurate way to measure the mass difference between the charged and neutral $\Xi$ particles.

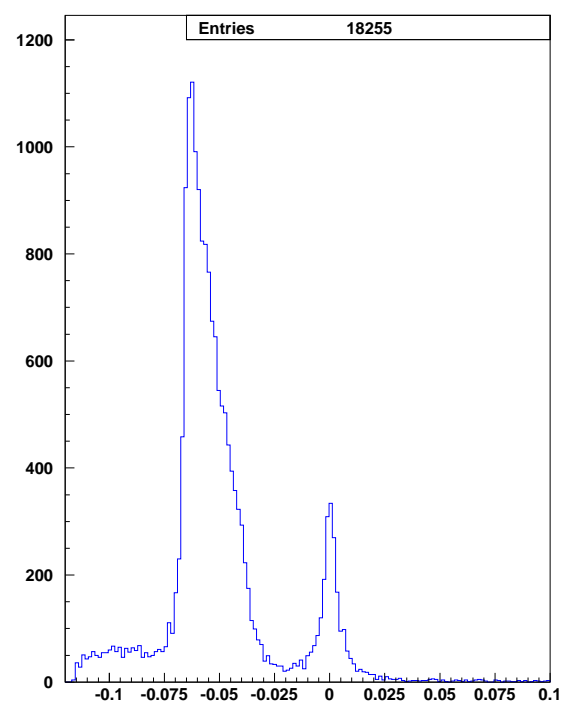

Figure 3. Preliminary results from the data obtained in the KTeV run of 1999 using the analysis techniques developed for the $\mathrm{KTeV} 1997$ data sample 11.13,14 yields more than $2100 \Xi^{0}$ beta decays (small peak centered on zero), here the horizontal axis is the mass of the reconstructed $\Sigma^{+}$(i.e. $\mathrm{p} \pi^{0}$ mass) subtracting its known value $1.189 \mathrm{GeV} / \mathrm{c}^{2}$. The peak on the left has been identified as several different background [14].

\section{First results from $\mathrm{KTeV}-99$}

Data from a new run of $\mathrm{KTeV}$ in 1999 with improved triggers, data acquisition and beam intensity is available for analysis. The new run is expected to yield a three fold increase in $\Xi^{0}$ beta decays, see figure 3, and a two fold increase in $\Xi^{0}$ muonic decays, see figure 1 . The circle points are

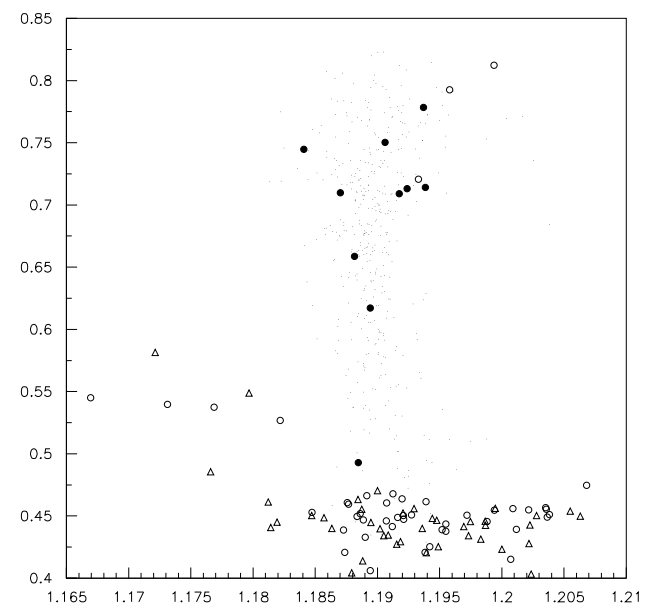

Figure 4. Preliminary results from the $\mathrm{KTeV}$ run in 1999 yields more $\Xi^{0}$ muonic decays. Here both axis are in units of $\mathrm{GeV} / \mathrm{c}^{2}$, on the horizontal is plotted the invariant mass of the $\mathrm{p} \pi^{0}$, and the vertical the mass $\left(\pi^{+} \mu^{-} \pi^{0}\right)$, from the dominant Kaon backgrounds: either $\mathrm{K}^{0} \rightarrow \pi^{+} \pi^{0} \pi^{-}$where $\pi^{-} \rightarrow \mu^{-} \bar{\nu}$ or $\mathrm{K} \mu 4$.

those events that satisfy the selection cuts except for the mass analysis of the two axis and $\mathrm{p}_{t}^{2}$ of the reconstructed $\Xi^{0}$, and the triangle points are the opposite charge sign analysis. There are far less anti $\Xi^{0}$ muonic decays expected in this wrong charge sign analysis, due to a $10 \mathrm{x}$ suppression of anti $\Xi^{0}$ at production. The horizontal band at the 
bottom are identifiable as the mentioned Kaon background decays, and the vertical band centered on $1.189 \mathrm{GeV} / \mathrm{c}^{2}$ are the $\Xi^{0}$ muonic decay events, the filled in data points are those that satisfy the kinematic cuts from the 1997 blind analysis $\left[\mathrm{p}_{t}^{2}<0.0008(\mathrm{GeV} / \mathrm{c})^{2}\right.$ and various mass cuts]. The small dots are the signal Monte-Carlo for the final event selection method. It was decided that our blind analysis from the 1997 data was sufficiently strong and straight forward enough that we did not need to make the 1999 analysis blind, but it could be applied directly to the new data set. This new $\mathrm{KTeV}$ data set should be calibrated by the end of 2000 and improved branching ratio, form factors, lifetime and possibly mass measurement within a year afterwards, depending on manpower and computer resources.

\section{Conclusion}

The hyperon program in $\mathrm{KTeV}$ has observed both semi-leptonic decays of the $\Xi^{0}$. A high statistics sample of the beta decay was used for measuring its branching ratio and form factors. Furthermore, the observation of the muonic decay of the neutral Cascade was observed with enough data to measure its branching ratio, but more data from the 1999 run is now available to improve this measurement. Furthermore, the $\Xi^{0}$ normal mode decay was also available to improve the mass and life-time values for this particle, important for $\mathrm{SU}(3)$ symmetry tests as well as improving the form factor measurements.

\section{REFERENCES}

1. A. Garcia and P. Kielanowski, The Beta Decay of Hyperons, Lecture Notes in Physics, 222, Springer-Verlag, Berlin, 1985.

2. M. Aguilar-Benitez et al., Phys. Lett. B 239 (1990) 1.

3. J. Lindquist et al., Phys. Rev. Lett. 27 (1971) 612, and Phys. Rev. D 16 (1977) 2104.

4. S. Y. Hsueh et al., Phys. Rev. D 38 (1988) 2056.

5. M. Bourquin et al., Z. Phys. C 21 (1983) 1, and Z. Phys. C 21 (1983) 37.
6. K. Arisaka et al., KTeV design report, Fermilab report FN-580, June 1992.

7. N. Solomey et al., "A Proposal for Hyperon Physics at KTeV", Univ. of Chicago note EFI 93-25, April 1993.

8. A. A. Affolder (N. Solomey advisor), "A Monte-Carlo Study of the $\Xi^{0} \rightarrow \Sigma^{+} e^{-} \bar{\nu}_{e}$ Decay in KTeV", Bachelor's Thesis at the Univ. of Chicago, May 1996.

9. A. Alavi-Harati et al., "Observation of the Decay $\Xi^{0} \rightarrow \Sigma^{+} \mu^{-} \bar{\nu}_{\mu} ", \mathrm{KTeV}$ internal report 547, August 1998.

10. V. Linke, Nucl. Phys. B 12 (1969) 669.

11. N. Solomey, "Recent Results in Weak Hyperon Decays," in Kaon Physics, ed. J.L. Rosner and B.D. Winstein (Chicago: University of Chicago Press, forthcoming), chap. 44.

12. A. Alavi-Harati et al., Observation of the Decay $\Xi^{0} \rightarrow \Sigma^{+} e^{-} \bar{\nu}_{e}$, Phys. Rev. Lett. 82 (1999) 3751-3754.

13. A. Alavi-Harati (A. Erwin advisor), "Observation and Branching Fraction Measurement of $\Xi^{0} \rightarrow \Sigma^{+} e^{-} \bar{\nu}$ at KTeV/E799-II, Fermilab", Ph.D. Thesis at the Univ. of Wisconsin, Oct. 1999.

14. S. Bright (R. Winston advisor), "First Measurement of Form Factors of the Beta Decay of the XI Hyperon", Ph.D. Thesis at the Univ. of Chicago, June 2000.

15. N. Solomey, Nucl. Instrum. and Methods A419 (1998) 637-641.

16. Particle Data Group, C. Caso et al., Eur. Phys. J. C3 (1998) 1.

17. M. Francis (E. Monnier advisor), "Measuring the Mass of the $\Xi^{0}$ in $\mathrm{KTeV}$ ", Undergraduate Thesis work at the Univ. of Chicago, August 1998.

18. V. Fanti et al., Eur. Phys. J. C 12 (2000) 6976.

19. C. Pietrykowski (N. Solomey advisor), "Study of $\Xi^{0}$ Decays for an Improved Lifetime Measurement with KTeV", Undergraduate Thesis work at the Univ. of Chicago, August 1999. 